in vivo $32: 1463-1471(2018)$

doi:10.21873/invivo.11400

\title{
Clinical and Radiological Mid- to Long-term Outcomes Following Ankle Fusion
}

\author{
INES E. GAEDKE ${ }^{1,2}$, ULRICH WIEBKING ${ }^{1,2}$, PADHRAIG F. O'LOUGHLIN ${ }^{3}$, \\ CHRISTIAN KRETTEK ${ }^{2}$ and RALPH GAULKE ${ }^{1,2}$ \\ ${ }^{1}$ Section Upper Extremity, Foot- and Rheuma Surgery, Trauma Department, \\ Medical School Hannover (MHH), Hannover, Germany; \\ ${ }^{2}$ Trauma Department, Medical School Hannover (MHH), Hannover, Germany; \\ ${ }^{3}$ Mater Hospital, Cork, Ireland
}

\begin{abstract}
Background: The aims of this study were to establish the likelihood of additional surgery after ankle fusion, determine the interval for developing osteoarthrosis in the ipsilateral subtalar or Chopart joints, and evaluate its clinical relevance. Material and Methods: A retrospective clinical and radiological study with a minimum follow-up of 24 months was performed. Short-Form 36 Heath Survey, Foot Function Index, American Orthopaedic Foot and Ankle Society Score (AOFAS) and a visual analog scale (VAS) were used to evaluate pain level and quality of life in at least 62 adult patients. Results: A total of $57 \%$ of our patients developed osteoarthrosis in at least one of the related joints and $28 \%$ of them required additional surgery due to pain. Patients who received workers' compensation had significantly lower AOFAS and higher VAS pain values. Conclusion: More than half of the study cohort developed osteoarthrosis in the related joints after ankle fusion, but fewer than one-third required further joint fusion surgery as a consequence.
\end{abstract}

Despite the emergence of total ankle arthroplasty, ankle fusion is still considered the gold standard for the treatment of severe osteoarthrosis of the ankle joint. It achieves significant pain relief in the majority of patients $(1,2)$. However, consequent overloading of the subtalar and

This article is freely accessible online.

Correspondence to: Professor Dr. med. Ralph Gaulke, Leiter der Sektion Obere Extremität, Fuß-und Rheumachirurgie, Unfallchirurgische Klinik, Medizinische Hochschule Hannover, Carl-Neuberg-Str. 1, D-30625 Hannover, Germany. E-mail: gaulke.ralph@mh-hannover.de

Key Words: Ankle fusion, osteoarthrosis, insurance status, revision surgery, long-term follow-up.
Chopart joints is known to cause secondary degenerative changes. This may cause pain and lead to further surgery with loss of hindfoot mobility.

Several studies have evaluated the mid- and long-term functional outcome after ankle fusion (1-7). Some studies compared the outcome after ankle fusion and arthroplasty (811). Good results were found in the majority of cases with high rates of patient satisfaction $(1,5,12)$. Nevertheless, Ebalard et al. reported that $84 \%$ of patients complained of pain after a minimum follow-up of 10 years (13). In other studies, the prevalence of osteoarthrosis ranged from $24-100 \%$ in the subtalar joint and from $18-77 \%$ in the Chopart joints (14).

The incidence of osteoarthrosis in adjacent joints after ankle fusion, as well as the most common interval within which it develops with long-term follow-up, is still unknown. Equally, the correlation between radiological findings and functional limitation and pain remains unclear. Fuchs et al. did not find a correlation between quality of life and the radiological grade of osteoarthrosis in the subtalar and Chopart joints (3).

The non-union rate after ankle fusion is reported to range between $1-16 \%(6,7,12,15)$. Obesity and nicotine abuse have been cited as risk factors for non-union in the current literature $(16,17)$. However, other studies do not fully corroborate these findings. Ebalard et al. did not find any correlation between non-union rate and body mass index (BMI) (13). Collman et $a l$. reported a trend towards non-union in obese patients (16). Previous studies have shown that insurance status also has an impact on results after surgery. Compared to Public Health Insurance, Workers Compensation seems to be a predictor for inferior outcomes (18).

The current investigators sought to evaluate how many patients developed osteoarthrosis in adjacent joints after ankle fusion, how long it took to develop osteoarthrosis, and how many of these patients required secondary surgery as a result. 


\section{Materials and Methods}

This retrospective clinical and radiological study was performed with approval of the local Ethic and Research Committee (No. 6927). All adult patients treated with ankle fusion surgery at the Trauma Department of the Medical School Hannover (MHH) between January 1998 and March 2015 were included in the study. The minimum follow-up was 2 years post-surgery.

Surgery was performed by senior orthopedic foot and ankle surgeons. Non-operative treatment, such as physical therapy, shoe modification and analgesia, had been tried prior to surgery for all patients.

Exclusion criteria included patients less than 18 years of age, any fusion procedure for other joints of the ipsilateral foot prior to ankle fusion, dementia, Korsakov syndrome, complete inactivity due to reasons other than the foot (for example, stroke) and language barriers.

Quality of life was evaluated using the Short Form 36 (SF-36), Foot Function Index (FFI), Hannover-Score, and the American Orthopaedic Foot and Ankle Society-Score (AOFAS) (19-22). Furthermore, the patients were asked four questions via a visual analog scale (VAS; scale 1-10): (i) How satisfied with your quality of life were you before the ankle fusion? (ii) How satisfied are you at follow-up? Rate your pain from 1 (no pain) to 10 (worst pain) on the VAS before (iii) and after (iv) surgery. The insurance status of each patient at the time of surgery was noted.

Clinical examination was performed by IG under supervision of RG and UW. It included a semi-quantitative analysis of the weightbearing pattern at the plantar aspect of the foot using a mirror-table (Figure 1); range-of-motion (ROM) testing of the hip, knee, subtalar and Chopart joints; measurement of the angle of the fused ankle joint; pain on pressure and translational stress; stability of the tarsal and tarsometatarsal joints; soft-tissue complaints; scar assessment; sensitivity to touch; and assessment of skin vascularity.

Dorsoplantar, lateral plain radiographs of the weightbearing foot, an oblique view of the foot, and anterior-posterior and lateral views of the ankle were performed. These were compared to pre-operative radiographs with regard to the development or progression of osteoarthrosis of the subtalar and Chopart joints. Union was defined as radiographic evidence of fusion at last follow-up.

Degenerative changes in the adjacent subtalar and Chopart joints were rated using the Kellgren-Lawrence Score (Table I). All patients were scored twice. Osteoarthrotic changes noted on plain radiographs, taken within an interval of 3 months pre-and postsurgery, were compared to radiographs taken at follow-up. All patients scoring grade two or higher were deemed to have osteoarthrosis. Using these two values for each patient, development and progression of osteoarthrosis at those adjacent joints was stratified.

Wilcoxon rank-test was used to assess whether the VAS scores related to pain and patient satisfaction had improved significantly preto postoperatively. In order to determine how many years after ankle fusion the subtalar and Chopart joints become arthritic, and to analyze the duration of pain relief after surgery, the study population was divided into four groups with regard to the follow-up: Group 1: Follow-up at 2 to 4 years; group 2: $>4$ to $<8$ years, group 3: $>8$ to $<12$ years, and group 4: $>12$ years. A cross table with a Chi-QuadratFishers test was used to compare the incidence of osteoarthrosis of adjacent joints between the groups and to compare the risk factors smoking and overweight (BMI $>25 \mathrm{~kg} / \mathrm{m}^{2}$ ) for non-union. A one- factor analysis of variance was performed to determine whether the mean quality of life and the mean AOFAS scores were different between the groups.

\section{Results}

Enrolment and patient details. Between January 1998 and March 2015, a total of 137 ankle fusions in 137 patients were performed at the Authors' university hospital. Twelve patients were excluded by exclusion criteria. Eleven patients had died and another 11 patients were lost to follow-up. A total of 103 patients were eligible for investigation; 85 of these had no further surgery of the treated foot and were invited to participate in the clinical and radiological examination. Six patients declined to participate. Sixty-three out of the remaining 79 patients took part in the clinical and radiological examination and answered the questionnaires. Sixteen patients only answered the questionnaires but declined clinical and radiological examination (Figure 2). From 18 patients who had undergone further surgery after ankle fusion, eight patients answered the questionnaires retrospectively, four patients declined to participate. Four patients underwent extended fusion and another two underwent lower leg amputation within 6 months following ankle fusion, which made questionnaires non-applicable. Radiographs of 10 patients who underwent secondary treatment were available for evaluation. Thus, along with 59 radiographs taken at the time of clinical examination, 69 patients were eligible for radiological followup examination. Radiographs of 86 patients were available for evaluation of bony union.

Fifty-eight $(56 \%)$ of the study participants were males, 45 $(44 \%)$ were females. The mean age at time of surgery was 53 (range=23-80) years. The mean BMI at surgery was 29 (range=20-49; $\mathrm{SD}=5.5$ ) $\mathrm{kg} / \mathrm{m}^{2}$. At the time of surgery, nine (9\%) patients suffered from diabetes and 38 were smokers (37\%). The mean follow-up time was 8.6 (range=0-19) years. The reason for ankle fusion was post-traumatic osteoarthrosis in the majority of cases (81\%). Gout-related arthritis, osteochondritis dissecans, degenerative osteoarthrosis, rheumatoid arthritis, septic arthritis, flesh-eating disease, club foot, Wegener's disease, and chondromatosis were other indications (Table II).

Nicotine abuse and being overweight (BMI $>25 \mathrm{~kg} / \mathrm{m}^{2}$ ) were not significant risk factors for non-union in this study. From 11 patients who developed a non-union, four were smokers, median body mass index (BMI) was 28 (range=20-42) $\mathrm{kg} / \mathrm{m}^{2}$, and one patient had diabetes. Thirty out of 75 patients with bony fusion were smokers, median BMI was 28 (range=20-44) $\mathrm{kg} / \mathrm{m}^{2}$, and seven patients suffered from diabetes.

Patient outcome scores and questionnaires. An AOFAS was completed in 62 patients, with the mean value being 62 (range $=12-92$ ). The mean value was significantly lower for the 


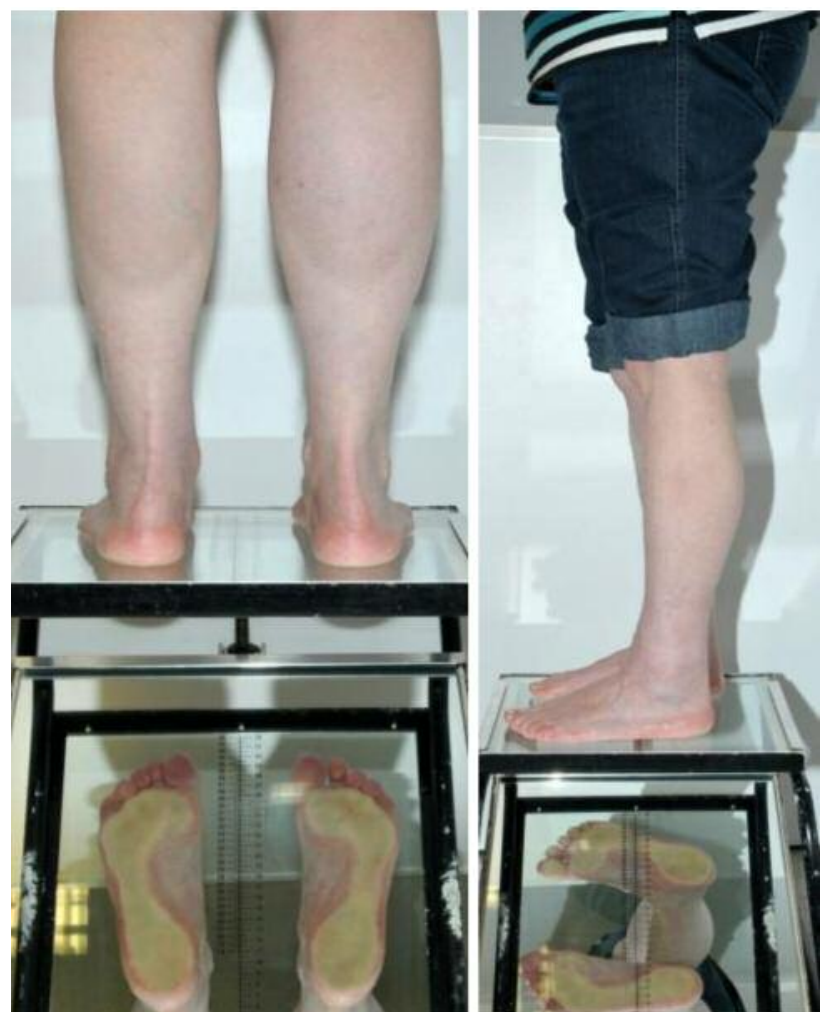

Figure 1. Patient standing on mirror-table.

group with $(n=39)$ than that without $(n=23)$ osteoarthrosis of the adjacent joints (58vs. 69; $p=0.005)$, and for the group with $(n=37)$ than that without $(n=25)$ secondary subtalar osteoarthrosis ( $57 v s .70 ; p=0.002)$, whilst the values between the groups with $(n=10)$ and without $(n=52)$ osteoarthrosis of the Chopart joint were not significantly different (57 vs. 63; $p=0.254$ ). This suggests that the negative effect on foot function caused by subtalar osteoarthrosis was greater than that caused by Chopart degeneration. The average SF-36 value was 57 (range=15-98). The mean value for mental health was 69 (range $=0-100$ ). For physical function, the mean value was 52 (range=5-100).

No significant differences were found between the four follow up-groups with regard to the VAS score for pain and patient satisfaction, FFI, Hannover-Score, pain-free walking distance, incidence of osteoarthrosis at the subtalar, Chopart, or both joints, or the mean values for AOFAS-and SF-36 scores. There was a trend towards lower AOFAS scores and lower SF-36 scores for greater follow-up periods. VAS values for pain were significantly higher before than after surgery ( 7.2 vs. $\left.3.8 ; p=2 \times 10^{-6}\right)$.

The questionnaires, which were completed retrospectively, were not taken into account in the calculations above, as it was felt by the Authors that these data should be treated separately for several reasons. Firstly, six out of eight patients who had completed the questionnaires retrospectively underwent arthrodesis of an adjacent joint within 4 years following their initial ankle arthrodesis. The other two patients required additional surgery at 11 and 16 years following their index procedure. Therefore, the impact of these questionnaires on the overall results would have varied from no or a minor impact within groups 2, 3 and 4 to a significant impact on follow-up group 1 . Secondly, it was felt that it would be inappropriate to compare retrospective data directly to the other questionnaires as it is difficult for patients to recall their pain level, satisfaction or walking ability several years post-surgery.

All eight patients were retrospectively asked to recall their experience from 4 weeks before they underwent additional surgery. The mean VAS for pain before and after surgery did not differ significantly amongst these patients (7.6 vs. 7.2, $p=0.785$ ). The reported pain-free walking distance was less than $500 \mathrm{~m}$ for six patients and between 500 and $1000 \mathrm{~m}$ for the other two. Only two patients stated their satisfaction post-surgery was 3 or lower. If these retrospective questionnaire data were taken into account, the average VAS for pain post-surgery would be higher than amongst those patients who stated their real-time pain (4.2 vs. 3.8).

Postoperative alignment. With regard to the sagittal plane, 43 out of $63(68 \%)$ ankles were fused in a neutral position $\left(90^{\circ}\right), 17$ out of $63(27 \%)$ in a plantarflexed position and three out of $63(5 \%)$ in a dorsiflexed position.

With regard to the coronal plane, $22(35 \%)$ ankles healed in $5^{\circ}$ of valgus and 21 ankles $(33 \%)$ fused in a neutral $\left(0^{\circ}\right)$ position. An increased hindfoot valgus of $10^{\circ}$ was found in five $(8 \%)$, of $15^{\circ}$ in three $(5 \%)$, and of $20^{\circ}$ in one patient (2\%). Seven ankles $(11 \%)$ were noted to have fused in $5^{\circ}$ of varus and 4 ankles $(6 \%)$ fused in $10^{\circ}$ of varus.

The mean subtalar ROM of the treated foot, with regard to supination and elevation was $18^{\circ}$ (range $=0-30^{\circ}$; median $\left.=15^{\circ}\right)$ compared to $29^{\circ}\left(\right.$ range $=15-30^{\circ} ;$ median $=30^{\circ}$ ) on the contralateral side. The mean ROM for extension and flexion of the chopart joint was $20^{\circ}$ (range $=5-35^{\circ}$; median $=20^{\circ}$ ) on the treated side compared to $18^{\circ}$ (range $=5$ $30^{\circ}$; median $=20^{\circ}$ ) of the contralateral Chopart joint.

Weight-bearing analysis. Assessment of the weight-bearing pattern of the operated foot, utilizing a mirror-table, revealed a swollen hindfoot in $55(87 \%)$ patients, a normal longitudinal arch in $52(83 \%)$, pes planus in three $(5 \%)$, splay and flatfoot in three $(5 \%)$ and pes cavus in five $(8 \%)$.

Vascularization and ROM. Vascularization of the skin was normal in all feet, whilst 16 out of 63 (25\%) patients had decreased skin sensitivity, mostly in the area around the 
Table I. Kellgren-Lawrence score.

\begin{tabular}{|c|c|c|c|}
\hline Factor & Feature & Score & \\
\hline \multirow[t]{3}{*}{ Osteophytes } & None & 0 & \\
\hline & Obvious & 1 & \\
\hline & Large & 2 & \\
\hline \multirow[t]{4}{*}{ Joint space } & Not narrowed & 0 & \\
\hline & Obviously narrowed & 1 & \\
\hline & Definitely narrowed & 2 & \\
\hline & Markedly narrowed & 3 & \\
\hline \multirow[t]{4}{*}{ Sclerosis } & None & 0 & \\
\hline & Small & 1 & \\
\hline & Small with cysts & 2 & \\
\hline & Severe with cysts & 3 & \\
\hline \multirow{4}{*}{ Deformity } & None & 0 & \\
\hline & Small & 1 & \\
\hline & Severe & 2 & \\
\hline & & Grade & Points \\
\hline \multirow[t]{5}{*}{ Feature } & Any degenerative changes & 0 & 0 \\
\hline & Doubtful narrowing of joint space and possible osteophytic lipping & 1 & $1-2$ \\
\hline & Definite osteophytes, possible narrowing of joints space & 2 & $3-4$ \\
\hline & $\begin{array}{l}\text { Moderate multiple osteophytes, definite narrowing of joints space, } \\
\text { some sclerosis and possible deformity of bone contour }\end{array}$ & 3 & $5-9$ \\
\hline & $\begin{array}{l}\text { Large osteophytes, marked narrowing of joint space, severe } \\
\text { sclerosis and definite deformity of bone contour }\end{array}$ & 4 & 10 \\
\hline
\end{tabular}

incision. No significant differences were found in terms of the ROM of the hip or knee joints when both lower limbs were compared in all patients.

Radiographic analysis. Plain radiographs showed an osseous ankle fusion in 75 out of $86(87 \%)$ patients, whilst $11(13 \%)$ had developed a non-union. Four out of these 11 patients did not have any clinical symptoms and did not require any further treatment. Another four patients suffered from pain, were unable to bear weight on the foot, and required revision ankle arthrodesis. After revision arthrodesis, all four patients achieved bony union. In the remaining three patients, the revision arthrodesis of the ankle joint was combined with a subtalar fusion for osteoarthrosis.

In 69 patients, radiographs were available at the time of surgery, at the time of arthrodesis of related joints, or at follow-up. In 44 (64\%) of these, radiological signs of subtalar osteoarthrosis (Kellgren-Lawrence Score $>2$ points) were found. Five of these suffered from pre-existing subtalar degeneration at the time of surgery. Twelve $(17 \%)$ out of the 69 patients showed radiological signs of osteoarthrosis in the Chopart joint. Two of these were pre-existing at the time of ankle fusion. At follow-up, 46 (67\%) out of 69 patients showed at least minor signs of osteoarthrosis in their subtalar or Chopart joints, or even in both. The majority of osteoarthrosis in related joints arose after ankle fusion (39/46,
Table II. Indications for ankle fusion.

\begin{tabular}{lc}
\hline Indication & $\mathrm{n}(\%)$ \\
\hline Post-traumatic osteoarthritis & $83(81 \%)$ \\
Gout-related arthritis & $6(6 \%)$ \\
Degenerative osteoarthritis & $3(3 \%)$ \\
Osteochondrosis dissecans & $3(3 \%)$ \\
Rheumatoid arthritis & $2(2 \%)$ \\
Septic arthritis & $2(2 \%)$ \\
Flesh-eating disease & $1(1 \%)$ \\
Clump foot & $1(1 \%)$ \\
Chondromatosis & $1(1 \%)$ \\
Wegners disease & $1(1 \%)$ \\
\hline Total & $103(100 \%)$ \\
\hline
\end{tabular}

$85 \%) ; 39 / 69(57 \%)$ of the patients developed osteoarthrosis of at least one related joint after ankle fusion (Table III).

Only 11 out of 39 patients (28\%) who developed osteoarthrosis in the related joints after ankle fusion needed additional surgery. Sixteen out of 39 (41\%) degenerative subtalar or Chopart joints were found within 4 years, five (13\%) after $4-8$ years, and $18(46 \%)$ after more than 8 years after surgery. Eight out of $16(50 \%)$ patients with early onset 


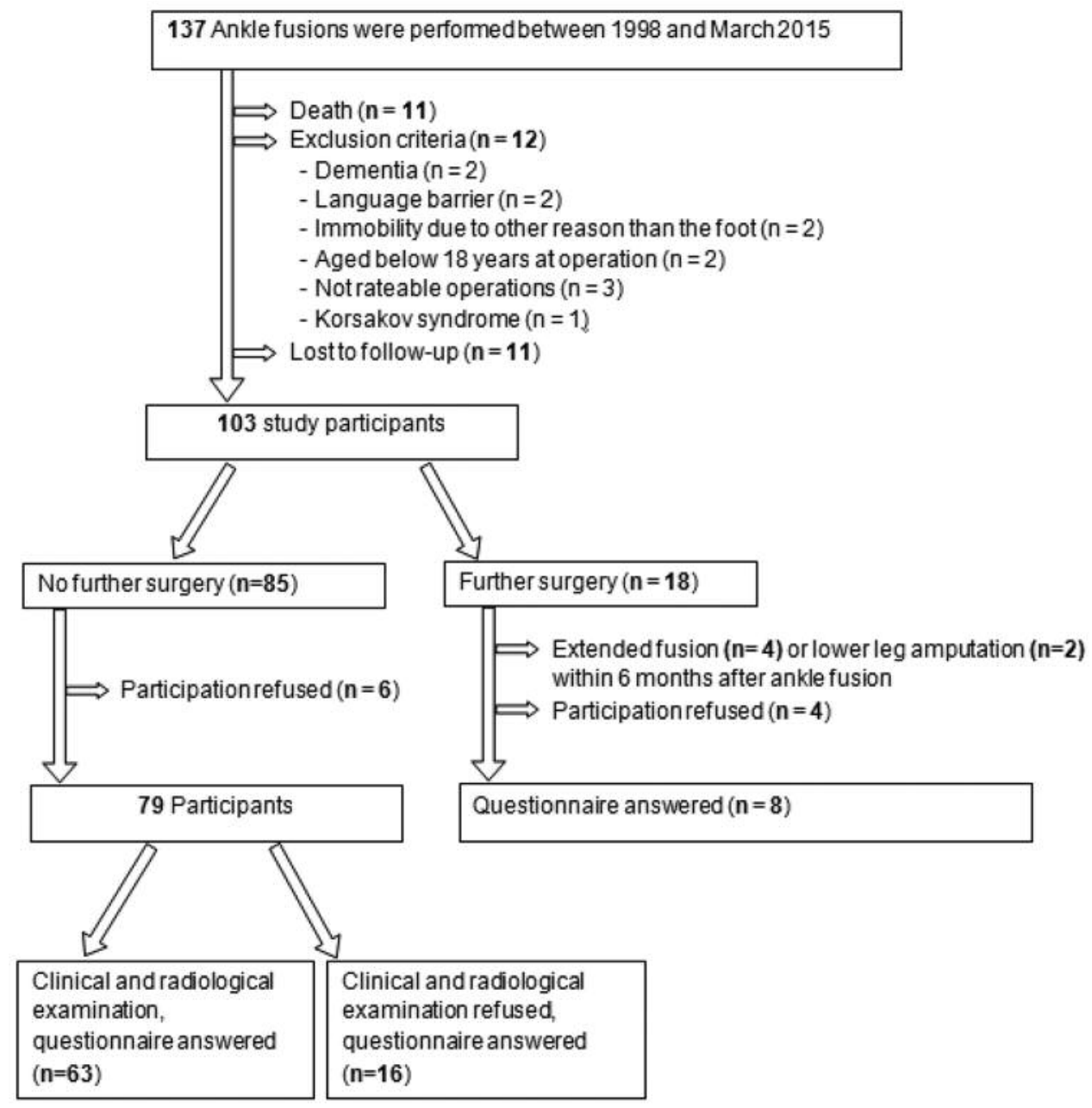

Figure 2. Study participation flowchart.

of related osteoarthrosis (within 4 years), but only three out of $18(17 \%)$ with late onset needed further surgery (Table IV, Figure 3). Two of the patients who suffered from pre-existing osteoarthrosis in the subtalar joint at the time of ankle fusion required additional surgery after 8 and 11 years.

The follow-up interval had no significant influence on the incidence of osteoarthrosis in related joints $(p=0.124)$. No evidence of stress fractures was found on the radiographs.

Influence of insurance status. No correlation $(p=0.899)$ was found between the insurance status of the patient and the union rate. By contrast, patients who received Workers' Compensation were found to have significantly lower subjective satisfaction scores: AOFAS scores were significantly lower (53.5 vs. 64.7; $p=0.017$ ) and postoperative VAS pain values were significantly higher ( $4.1 v s .2 .9 ; p=0.018)$ than in patients covered by private or public health insurance.
Additional surgery. At follow-up, 18 out of the 103 patients included in the study had required further surgery. Sixteen patients $(16 \%)$ underwent an extended fusion of related joints. Another two (2\%) patients underwent lower-leg amputation. At the time of follow-up, another three patients decided to have further surgery: Subtalar fusion, pan-talar fusion, and lower leg amputation were performed once each. Overall $21(20 \%)$ patients required further surgery between $0-16$ years after ankle fusion.

Twenty-one out of 103 (20\%) patients underwent extended fusion surgery $(n=18)$ or lower leg amputation $(n=3)$ after ankle fusion. Thirteen out of the 18 subtalar-or Chopart fusions and two lower leg amputations were performed within 4 years after initial ankle fusion. Five subtalar with/without Chopart fusions and one lower leg amputation were required at greater than 8 years post-ankle fusion (Figure 4). 


\section{Discussion}

This study sought to evaluate the clinical outcomes of patients with ankle fusion, and to establish how many patients developed osteoarthrosis in adjacent joints, how long it took to develop osteoarthrosis, what the clinical impact was and how many of these patients required secondary surgery as a result.

The SF-36 values in this study cohort are comparable to those published by Dalat et al. (9), showing limitation in physical function but good scores in terms of mental health.

The average AOFAS score of 62 in this cohort at 9.6 (5.6 $\mathrm{SD})$ years of follow-up is slightly lower compared to other reported results by Braito et al. (8) and Hendrickx et al. (1). Braito et al. reported a global AOFAS score of 68.3 in 16 ankle fusions at a mean follow-up of 4.4 years and Hendrickx et al. reported a mean value of 67 in 60 patients at a mean follow-up of 9 (SD 4.1) years. The difference in the current study's findings may be caused by the longer follow-up period, as 27 of the ankle fusions were reviewed at more than 10 years post-surgery, as well as the high rate of post-traumatic osteoarthrosis with soft-tissue trauma and the age differences between the studied cohorts.

The etiology for osteoarthrosis of the ankle joint reported by Hendrickx et al. was principally trauma (87\%) (1). Braito et al. included primary and post-traumatic osteoarthrosis (8). The patient cohort in the study by Hendricks et al. was younger than that of the current study with $51 \%$ less than 50 years of age at the time of surgery (1). In the current study, only $40 \%$ were younger than 50 years at surgery. A negative correlation between AOFAS score and follow-up after surgery was found by Houdek et al. who compared 2- , 5and 10-year postoperative AOFAS scores after contralateral fusions (81.1, 76.3, and 72.2, respectively) (23). Nevertheless, those scoring systems used revealed that the majority of the patients were satisfied with their clinical outcome. It should be noted, however, that a few patients had poor results with low AOFAS scores and high VAS regarding pain. Neuropathic pain after soft-tissue trauma, painful scars, reduced weight-bearing area of the plantar aspect of the foot due to suboptimal arthrodesis position and osteomyelitis are possible reasons for persisting complaints $(5,24,25)$. One aim of the current study was to determine how long clinical benefit from ankle fusion endures. No significant difference was seen amongst the four subgroups according to clinical and radiological follow-up.

Some studies have described hypermobility of the midtarsal joints after ankle fusion compared to the unaffected side $(16,26)$. Conversely, Jackson et al. (27) and van der Plaat et al. (28) found decreased motion or no change in the ROM. Immobility of the fused ankle joint leads to a secondary overload of the tarsal joints, which may lead to instability causing degenerative changes over time (29). Our
Table III. Distribution of KLS of the subtalar and Chopart joints among the studied patients.

\begin{tabular}{lcc}
\hline KLS & Subtalar joint & Chopart joint \\
\hline 0 & $0(0)$ & $0(0)$ \\
1 & $25(0)$ & $57(0)$ \\
2 & $36(2)$ & $11(2)$ \\
3 & $8(3)$ & $1(0)$ \\
4 & 0 & 0 \\
Osteoarthrosis total (KLS=2-4) & $44(5)$ & $12(2)$ \\
All patients (KLS 0-4) & $69(5)$ & $69(2)$ \\
\hline
\end{tabular}

KLS: Kellgren-Lawrence Score. Number of patients with pre-existing osteoarthrosis at time of ankle fusion shown in parentheses.

Table IV. Number of patients with degenerated related joints and need for surgery.

\begin{tabular}{lcccc}
\hline Follow-up, years & Subtalar OA & Chopart OA & Total & Surgery \\
\hline$<4$ & 16 & 3 & 16 & 8 \\
$4-8$ & 4 & 1 & 5 & 0 \\
$>8$ & $24(5)$ & $8(2)$ & $25(7)$ & $5(2)$ \\
$0-20$ & $44(5)$ & $12(2)$ & $46(7)$ & $13(2)$ \\
\hline
\end{tabular}

Number of patients with pre-existing osteoarthrosis (OA) at the time of ankle fusion in parenthesis.

study found only a very small difference in the ROM comparing the ipsi- and contralateral Chopart joints in every patient, as van der Plaat et al. described (28). Most of the current study's patients had reduced subtalar ROM compared to the opposite uninjured side. This was also reported by Lynch et al. (7), Said et al. (15) and Jackson et al. (27). Thomas et al. also found fore-and hindfoot motion measured while walking decreased after ankle arthrodesis (30).

In the current study, $32 \%$ of the feet were not fused in a neutral position and ROM was measured using a goniometer, which may have influenced the results. Nevertheless, patients with up to five degrees of ankle plantarflexion or dorsiflexion did not show an inferior outcome to those patients with neutral fusion position. Lynch et al. found no difference in the mean Mazur-Score comparing patients with neutral arthrodesis to those with plantar-or dorsiflexion (7). Ratliff et al. recommended the neutral position or a slight drop foot or calcaneal position in order to have the best outcome (31).

Our study did not find a correlation between the incidence of osteoarthrosis in the subtalar and Chopart joints and the time after surgery. We interpreted this to signal that degenerative changes of the related joints may appear at any time after fusion. Nevertheless, there was a bimodal trend towards developing arthrosis either in the first 4 years after 

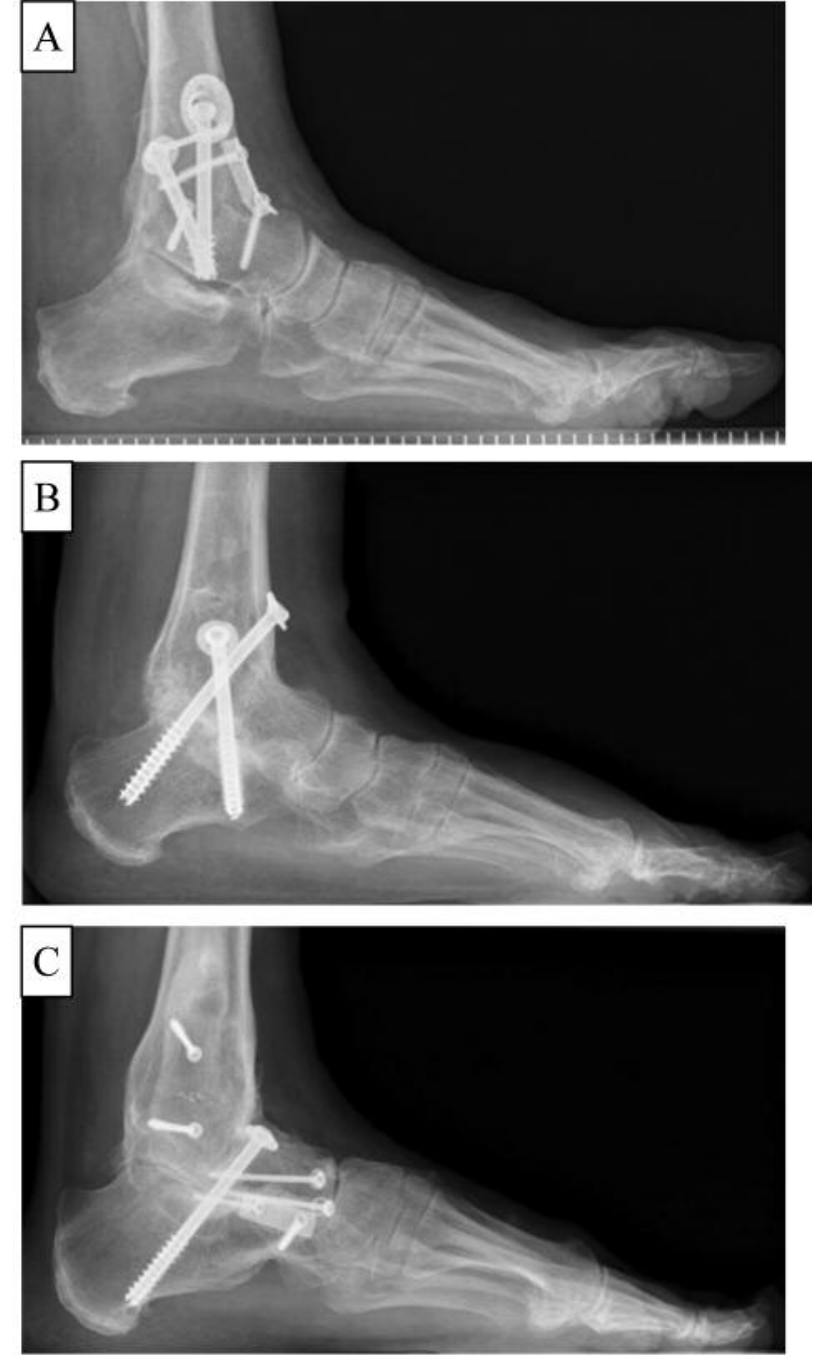

Figure 3. A: Ankle fusion. B: Ankle and subtalar fusion. C: Pantalar fusion.

fusion, which happened to most of the patients who answered the questionnaires retrospectively, or at 8 years and onwards. This trend can also be seen when looking at the period of time from ankle arthrodesis to extended fusion. Many patients required secondary treatment due to arthrosis in a related joint either within the first 4 years after fusion or greater than eight years. Patients with a follow-up of between 4 and 8 years showed the best results with regard to pain relief and quality of life. This might be explained by patients having become familiar with their new gait pattern whilst any osteoarthrosis of related joints caused by overload remained asymptomatic.

Patients should be informed about the possibility of developing painful degeneration of related joints soon after ankle fusion before the decision for ankle arthrodesis is made. Considering the patient retrospective questionnaire

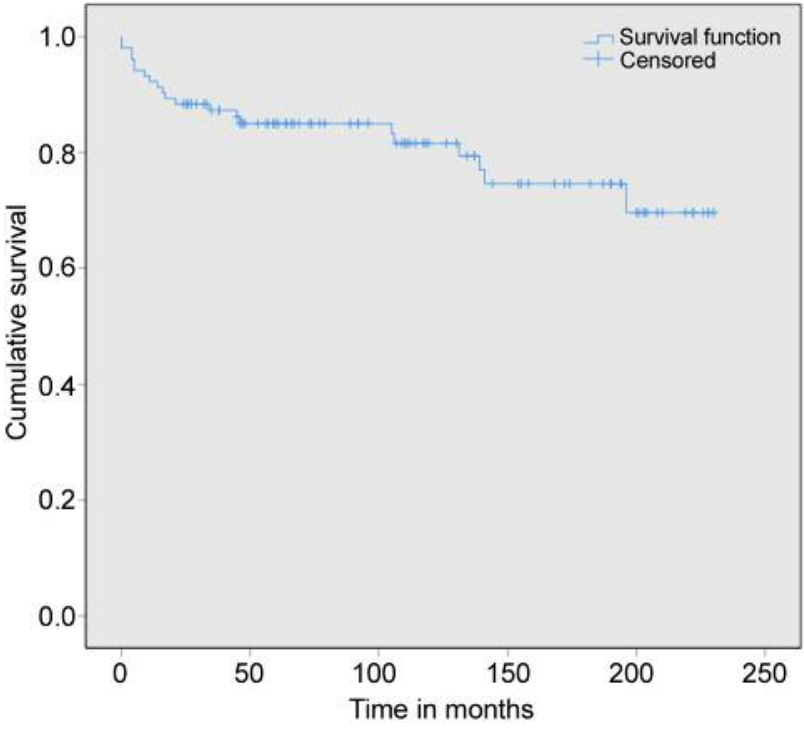

Figure 4. Kaplan-Meier survival analysis: Cumulative likelihood of requiring additional surgery after ankle fusion over time.

data, we recognize that reporting of pain was greater than the previously reported results.

At mid- to long-term follow-up, $57 \%$ of the patients in the study developed osteoarthrosis in related joints. This rate is higher than in most previous studies with shorter follow-up. Previous studies evaluated the incidence of osteoarthrosis in related joints at short- to mid-term follow-up. In their cohort of 72 patients, with a mean follow-up of 5.9 (range=4.8-7.8) years, Zwipp et al. reported radiographic evidence of osteoarthrosis after ankle arthrodesis in $35 \%$ of the subtalar and in $18 \%$ of the talonavicular joints. None of these patients underwent further fusion surgery. In that cohort ankle fusion was mainly (90\%) performed for post-traumatic arthrosis (6). Kopp et al. found osteoarthrosis in $24 \%$ of the subtalar joints at a mean of 7.3 (range $=2-20$ ) years after ankle arthrodesis. The etiology of ankle osteoarthrosis was trauma in $63 \%$ of the patients (12). Only Hendrickx et al. reported radiographic evidence of preoperative degeneration in $91 \%$ of the subtalar and in $77 \%$ of the talonavicular joints after a mean follow up of $9(\mathrm{SD}=4.1)$ years. The etiology for ankle osteoarthrosis was post-traumatic in most $(87 \%)$ of these cases (1).

In the current study cohort, $57 \%$ of the patients developed osteoarthrosis in the subtalar or Chopart joints, or both, but only $28 \%$ were treated with additional surgery. Other studies reported less or even no incidence $(0-9 \%$, follow-up $=5.9-8.5$ years) of additional surgery $(5,6,32)$. It is important to note that six patients in the current study underwent subtalar with/without Chopart fusion within 6 months of ankle fusion. Upon review, isolated ankle fusion was likely not the correct 
decision in these cases. In three cases, an additional subtalar fusion and in another case a pan-talar fusion would have been appropriate. In two other cases of primary ankle fusion in acute trauma surgery, the lower leg could not be salvaged due to the severity of the associated soft-tissue trauma. Seven $(8 \%)$ of this study's patients required additional surgery for nonunion. This non-union rate is within the reported range (6 to $16 \%)$ reported in the literature $(1,3,11,12,15)$.

The literature contains conflicting reports about the influence of nicotine abuse and obesity on osseous union in hindfoot fusion procedures. Easley et al. found a significant correlation between the non-union rate of subtalar fusion and smoking (33), while Joveniaux et al. did not (34). Ishikawa et $a l$. found the relative risk of developing a non-union after hindfoot fusion to be 2.7 times higher for smokers (17). Suda et al. found no correlation between BMI and the non-union rate in arthrodesis for septic arthritis of the ankle (35). Collman et al. reported a trend towards non-union in obese patients (16). The current study did not find nicotine abuse or elevated BMI to be significant risk factors for non-union of ankle fusion.

In the current study cohort, the subjective, but not the objective, outcome was inferior in patients covered by Workers' Compensation compared to those with private or public health insurance. This effect has been reported before for different conditions $(18,36)$.

The current study is limited by its retrospective design, a heterogenous cohort and the range of clinical indications for ankle fusion. It is difficult to compare, for example, the results of primary ankle fusion due to trauma with elective arthrodesis for degenerative osteoarthrosis. Furthermore, all ankle fusions were performed over a long period of time by different surgeons who used different approaches and fixation methods. The VAS for pain and satisfaction at time of surgery was evaluated retrospectively, which normally leads to lower pain-related values due to challenges for the patient in terms of recall. Eight patients who had already undergone additional fusion of related joints at follow-up were asked retrospectively. It is difficult for patients to remember their specific pain levels years after the event. This can lead to inaccurate data.

\section{Conclusion}

More than half of the study cohort developed osteoarthrosis in related joints after ankle fusion, but fewer than one-third required further joint fusion surgery as a consequence. Radiological findings were not strongly related to clinical outcome nor the experience of pain. Osteoarthrosis of the subtalar joint is common in patients after ankle fusion. Receiving Workers' Compensation tended to have a negative effect on the subjective but not on the objective outcomes of ankle fusion.

\section{Acknowledgements}

This study was financially supported by the KlinStrucMed program of the independent Else-Kröner-Foundation (Else-Kröner-Stiftung) for the clinical MD of IG.

\section{References}

1 Hendrickx RPM, Stufkens SAS, de Bruijn EE, Sierevelt IN, van Dijk CN and Kerkhoffs GMMJ: Medium-to long-term outcome of ankle arthrodesis. Foot Ankle Int 32(10): 940-947, 2011.

2 Takakura Y, Tanaka Y, Sugimoto K, Akiyama K and Tamai S: Long-term results of arthrodesis for osteoarthritis of the ankle. Clin Orthop Relat Res 361(4): 178-185, 1999.

3 Fuchs S, Sandmann C, Skwara A and Chylarecki C: Quality of life 20 years after arthrodesis of the ankle. A study of adjacent joints. J Bone Joint Surg Br 85(7): 994-998, 2003.

4 Kennedy JG, Hodgkins CW, Brodsky A and Bohne WH: Outcomes after standardized screw fixation technique of ankle arthrodesis. Clin Orthop Relat Res 447(6): 112-118, 2006.

5 Kerkhoff YRA, Keijsers NLW and Louwerens JWK: Sports participation, functional outcome, and complications after ankle arthrodesis: Midterm follow-up. Foot Ankle Int 38(10): 10851091, 2017.

6 Zwipp H, Rammelt S, Endres T and Heineck J: High union rates and function scores at midterm followup with ankle arthrodesis using a four screw technique. Clin Orthop Relat Res 468(4): 958-968, 2010.

7 Lynch AF, Bourne RB and Rorabeck $\mathrm{CH}$ : The long-term results of ankle arthrodesis. J Bone Joint Surg Br 70(1): 113-116, 1988.

8 Braito M, Dammerer D, Kaufmann G, Fischler S, Carollo J, Reinthaler A, Huber D and Biedermann R: Are our expectations bigger than the results we achieve? A comparative study analysing potential advantages of ankle arthroplasty over arthrodesis. Int Orthop 38(8): 1647-1653, 2014.

9 Dalat F, Trouillet F, Fessy MH, Bourdin M and Besse JL: Comparison of quality of life following total ankle arthroplasty and ankle arthrodesis: Retrospective study of 54 cases. Orthop Traumatol Surg Res 100(7): 761-766, 2014.

10 SooHoo NF, Zingmond DS and Ko CY: Comparison of reoperation rates following ankle arthrodesis and total ankle arthroplasty. J Bone Joint Surg Am 89(10): 2143-2149, 2007.

11 Haddad SL, Coetzee JC, Estok R, Fahrbach K, Banel D and Nalysnyk L: Intermediate and long-term outcomes of total ankle arthroplasty and ankle arthrodesis. A systematic review of the literature. J Bone Joint Surg Am 89(9): 1899-1905, 2007.

12 Kopp FJ, Banks MA and Marcus RE: Clinical outcome of tibiotalar arthrodesis utilizing the chevron technique. Foot Ankle Int 25(4): 225-230, 2004.

13 Ebalard M, Le Henaff G, Sigonney G, Lopes R, Kerhousse G, Brilhault $J$ and Huten D: Risk of osteoarthritis secondary to partial or total arthrodesis of the subtalar and midtarsal joints after a minimum follow-up of 10 years. Orthop Traumatol Surg Res 100(4): 231-237, 2014.

14 Ling JS, Smyth NA, Fraser EJ, Hogan MV, Seaworth CM, Ross KA and Kennedy JG: Investigating the relationship between ankle arthrodesis and adjacent-joint arthritis in the hindfoot. A systematic review. J Bone Joint Surg Am 97(9): 513-520, 2015. 
15 Said E, Hunka L and Siller TN: Where ankle fusion stands today. J Bone Joint Surg Br 60(2): 211-214, 1978.

16 Collman DR, Kaas MH and Schuberth JM: Arthroscopic ankle arthrodesis: factors influencing union in 39 consecutive patients. Foot Ankle Int 27(12): 1079-1085, 2006.

17 Ishikawa SN, Murphy GA and Richardson EG: The effect of cigarette smoking on hindfoot fusions. Foot Ankle Int 23(11): 996-998, 2002.

18 Peters S, Persson J, O'Loughlin P, Krettek C and Gaulke R: Long-term economical effects of isolated calcaneus fracture depending on insurance status, age, occupation and fracture type. Clin Res Foot Ankle 5(1): 1-7, 2017.

19 Button G and Pinney S: A meta-analysis of outcome rating scales in foot and ankle surgery: is there a valid, reliable, and responsive system? Foot Ankle Int 25(8): 521-525, 2004.

20 SooHoo NF, Shuler M and Fleming LL: Evaluation of the validity of the AOFAS clinical rating systems by correlation to the SF-36. Foot Ankle Int 24(1): 50-55, 2003.

21 McHorney CA, Ware JE Jr, Lu JFR and Sherbourne CD: The MOS 36-item Short-Form Health Survey (SF-36): Tests of data quality, scaling assumptions, and reliability across diverse patient groups. Medical Care 32(1): 40-66, 1994.

22 Budiman-Mak E, Conrad KJ and Roach KE: The Foot Function Index: a measure of foot pain and disability. J Clin Epidemiol 44(6): 561-570, 1991.

23 Houdek MT, Wilke BK, Ryssman DB and Turner NS: Radiographic and functional outcomes following bilateral ankle fusions. Foot Ankle Int 35(12): 1250-1254, 2014.

24 Labitzke R: Ankle arthrodesis using the cable technique. Oper Orthop Traumatol 17(4-5): 392-406, 2005.

25 Kolodziej L, Sadlik B, Sokolowski S and Bohatyrewicz A: Results of arthroscopic ankle arthrodesis with fixation using two parallel headless compression screws in a heterogenic group of patients. Open Orthop J 11(2): 37-44, 2017.

26 Morgan CD, Henke JA, Bailey RW and Kaufer H: Long-term results of tibiotalar arthrodesis. J Bone Joint Surg Am 67(4): 546-550, 1985.
27 Jackson A and Glasgow M: Tarsal hypermobility after ankle fusion-fact or fiction? J Bone Joint Surg Br 61(4): 470-473, 1979.

28 van der Plaat LW, van Engelen SJPM, Wajer QE, Hendrickx RPM, Doets KHC, Houdijk H and van Dijk CN: Hind-and midfoot motion after ankle arthrodesis. Foot Ankle Int 36(12): 1430-1437, 2015.

29 Suckel A, Muller O, Herberts T and Wulker N: Changes in Chopart joint load following tibiotalar arthrodesis: in vitro analysis of 8 cadaver specimens in a dynamic model. BMC Musculoskelet Disord 8:80: 1-9, 2007.

30 Thomas R, Daniels TR and Parker K: Gait analysis and functional outcomes following ankle arthrodesis for isolated ankle arthritis. J Bone Joint Surg Am 88(3): 526-535, 2006.

31 Ratliff AH: Compression arthrodesis of the ankle. J Bone Joint Surg Br 41(3): 524-534, 1959.

32 Strasser NL and Turner NS: Functional outcomes after ankle arthrodesis in elderly patients. Foot Ankle Int 33(9): 699-703, 2012.

33 Easley ME, Trnka HJ, Schon LC and Myerson MS: Isolated subtalar arthrodesis. J Bone Joint Surg Am 82(5): 613-624, 2000.

34 Joveniaux P, Harisboure A, Ohl X and Dehoux E: Long-term results of in situ subtalar arthrodesis. Int Orthop 34(8): 1199$1205,2010$.

35 Suda AJ, Richter A, Abou-Nouar G, Jazzazi M, Tinelli M and Bischel OE: Arthrodesis for septic arthritis of the ankle: risk factors and complications. Arch Orthop Trauma Surg 136(10): 1343-1348, 2016.

36 Buckley R, Tough S, McCormack R, Pate G, Leighton R, Petrie $\mathrm{D}$ and Galpin R; Operative compared with nonoperative treatment of displaced intra-articular calcaneal fractures: A prospective, randomized, controlled multicenter trial. J Bone Joint Surg Am 84(10): 1733-1744, 2002.

Received June 6, 2018

Revised June 20, 2018

Accepted June 28, 2018 